\title{
Análise epidemiológica de hanseníase no Brasil no período de 2016 a 2020
}

\author{
Epidemiological analysis of leprosy in Brazil from 2016 to 2020 \\ Análisis epidemiológico de la lepra en Brasil de 2016 a 2020
}

\section{Resumo}

Introdução: A hanseníase é uma doença crônica infecciosa relacionada a fatores sociais e econômicos, ainda muito presente no Brasil. Objetivo: O presente estudo teve como objetivo traçar o perfil epidemiológico da Hanseníase no Brasil, no período de 2016 e 2020. Métodos: Trata-se de um estudo epidemiológico descritivo, retrospectivo com abordagem quantitativa. Os dados foram obtidos por meio da consulta ao Departamento de Informática do Sistema Único de Saúde (DATASUS). Resultados: A Hanseníase ainda é um problema de saúde pública no país, visto que o Brasil é o maior responsável por casos da doença na América Latina, destacando-se as regiões Nordeste, Norte e Centro Oeste como as principais produtoras de novos casos. Em acréscimo, o gênero masculino é o mais acometido pela doença, já que ele desenvolve uma resposta imunológica menor quando comparado ao gênero feminino, afetando principalmente indivíduos economicamente ativos. Além disso, a falta de acesso à informação e a precariedade socioeconômica são fatores que potencializam a falta de tratamento e a disseminação da doença, com ênfase em sua forma multibacilar. Conclusão: Fica clara a necessidade da elaboração de um plano que incentive a detecção precoce e a redução das incapacidades causadas pela doença, por meio de ações de vigilância em saúde, visando o combate. Ademais, é de suma importância a adaptação da política de atenção à Hanseníase à realidade das regiões onde a incidência é maior, a fim de aumentar a adesão ao tratamento e, consequentemente, erradicar a doença.

Palavras-chave: Hanseníase; Epidemiologia; Doença infecciosa; Erradicação; Brasil. 


\begin{abstract}
Introduction: Leprosy is a chronic infectious disease related to social and economic factors, still very present in Brazil Objective: This study aimed to outline the epidemiological profile of leprosy in Brazil between 2016 and 2020.Methods: This is a descriptive, retrospective epidemiological study with a quantitative approach. Data were obtained by consulting the Department of Informatics of the Unified Health System (DATASUS). Results: Leprosy is still a public health problem in the country, as Brazil is the most responsible for cases of the disease in Latin America, with the Northeast, North and Midwest regions standing out as the main producers of new cases. In addition, males are the most affected by the disease, as they develop a lower immune response when compared to females, mainly affecting economically active individuals. In addition, the lack of access to information and socioeconomic precariousness are factors that increase the lack of treatment and the spread of the disease, with emphasis on its multibacillary form. Conclusion: The need to develop a plan that encourages early detection is clear. and the reduction of incapacities caused by the disease, through health surveillance actions, aimed at combating it. Furthermore, it is extremely important to adapt the leprosy care policy to the reality of regions where the incidence is higher, to increase adherence to treatment and, consequently, eradicate the disease.
\end{abstract}

Keywords: Leprosy; Epidemiology; Infectious disease; Eradication; Brazil.

\title{
Resumen
}

Introducción: La lepra es una enfermedad infecciosa crónica relacionada con factores sociales y económicos, aún muy presente en Brasil. Objetivo: Este estudio tuvo como objetivo delinear el perfil epidemiológico de la lepra en Brasil entre 2016 y 2020. Métodos: Se trata de un estudio epidemiológico descriptivo, retrospectivo con enfoque cuantitativo. Los datos se obtuvieron consultando al Departamento de Informática del Sistema Único de Salud (DATASUS). Resultados: La lepra sigue siendo un problema de salud pública en el país, ya que Brasil es el mayor responsable de casos de la enfermedad en América Latina, destacándose las regiones Nordeste, Norte y Medio Oeste como las principales productoras de nuevos casos. Además, los machos son los más afectados por la enfermedad, ya que desarrollan una menor respuesta inmune en comparación con las hembras, afectando principalmente a las personas económicamente activas. Además, la falta de acceso a la información y la precariedad socioeconómica son factores que aumentan la falta de tratamiento y la propagación de la enfermedad, con énfasis en su forma multibacilar. Conclusión: Es clara la necesidad de desarrollar un plan que incentive la detección temprana. la reducción de las incapacidades causadas por la enfermedad, a través de acciones de vigilancia de la salud, dirigidas a combatirla. Además, es de suma importancia adecuar la política de atención de la lepra a la realidad de las regiones donde la incidencia es mayor, para aumentar la adherencia al tratamiento y, en consecuencia, erradicar la enfermedad.

Palabras clave: Lepra; Epidemiología; Enfermedad infecciosa; Erradicación; Brasil.

\section{Introdução}

A hanseníase é uma doença crônica infecciosa, de evolução lenta, causada pelas bactérias Mycobacterium leprae e Mycobacterium lepromatosis (Ploemacher et al., 2020). Ela pode ser caracterizada por ter capacidade de gerar lesões nos nervos e na pele de seus portadores, além de ocasionar cegueira ou até mesmo perda de membros em alguns casos (Santos et al., 2020). É considerada uma das patologias mais antigas e é quase sempre relacionada com condições socioeconômicas ruins, desigualdade social e serviços de saúde com baixa efetividade (Lopes et al., 2021).

No ano de 2017, cerca de $13 \%$ dos mais de 200.000 casos mundiais de hanseníase ocorreram no Brasil, tendo uma distribuição bastante heterogênea (Pescarini et al., 2021). A discrepância do número de casos nas diferentes regiões do país se deve à grande extensão territorial e às desigualdades sociais, que tornam alguns lugares endêmicos, colaborando para que a doença seja um relevante problema de saúde pública (Ribeiro et al., 2018).

Dessa forma, a Organização Mundial da Saúde, em 1991, depois de estabelecer a poliquimioterapia como tratamento específico para a hanseníase, apresentou a proposta de eliminar a doença como um problema de saúde pública até o ano 2000 (Campos et al., 2018). Porém, o Brasil ainda não conseguiu alcançar a meta, estando em segundo lugar na relação de países com maiores números de novos diagnósticos, atrás somente da Índia. Na atualidade, o país possui mais de $90 \%$ dos casos de toda América Latina, algo bastante preocupante para as agências de saúde (Souza et al., 2020).

Dito isso, destacam-se os desafios para controlar a hanseníase, nos quais estão inclusos a continuidade da transmissão dos agentes etiológicos, a dificuldade na vigilância do contato dos pacientes e o conhecimento limitado sobre a transmissão da 
doença. Sendo assim, existem políticas de manejo da patologia, que para serem efetivas, necessitam da identificação das pessoas com maior risco de adoecer (Nitsuma et al., 2021).

Portanto, esse estudo se justifica devido à importância de análises sobre as características epidemiológicas da hanseníase, já que são necessárias para entender os fatores que colaboram para manutenção do número de casos do país nos últimos anos e podem contribuir para o controle da doença. Dessa forma, o presente estudo teve como objetivo traçar o perfil epidemiológico da Hanseníase no Brasil, no período de 2016 e 2020.

\section{Metodologia}

Trata-se de um estudo epidemiológico descritivo, retrospectivo com abordagem quantitativa. Os dados foram obtidos por meio da consulta ao Departamento de Informática do Sistema Único de Saúde (DATASUS), no endereço eletrônico (http://www.datasus.gov.br) nas seguintes bases de dados: Sistema de Informação de Agravos de Notificação. O levantamento de dados ocorreu nos meses de setembro e outubro de 2021. Para evitar erros de retardo de notificação, optou-se por analisar os dados disponíveis até 2020, último ano em que constavam os dados completos.

A população de interesse é constituída por todos os casos de hanseníase no Brasil, diagnosticados e registrados no período de 2016 a 2020.

Foram analisados os seguintes parâmetros: faixa etária, gênero, raça, escolaridade, casos em gestante, região de residência, UF de residência, município de residência, classificação operacional, forma clínica, graus de incapacidade e tipo de saída (cura, abandono ou óbito).

Os dados foram inicialmente tabulados no Microsoft Office Excel e foram tratados estatisticamente (em termos de média, correlações e números absolutos), por meio do programa Statistical Package for the Social Sciences (SPSS) versão 20.0.

Por se tratar de um banco de domínio público, não foi necessário submeter o projeto ao Comitê de Ética em Pesquisa.

\section{Resultados e discussão}

No período estudado, as regiões que registraram maior prevalência dos casos de Hanseníase são, em ordem decrescente, Nordeste (67070 casos), com destaque para o Maranhão (11,19\% dos casos), Norte (34226 casos), no qual há maior relevância no Pará (9,71\% dos casos) e Centro-Oeste (31623), com maior incidência no Mato Grosso (13,89\% dos casos). Em sequência, com índices menores da doença, estão as regiões Sudeste (21563 casos) e Sul (5034 casos), como mostra a Tabela 1. 
Tabela 1 - Relação dos números absolutos e proporções de casos de Hanseníase, pela UF de notificação e ano de diagnóstico dos casos de Hanseníase no Brasil, entre 2016 e 2020.

\begin{tabular}{|c|c|c|c|c|c|c|c|c|c|c|c|c|}
\hline \multirow{2}{*}{ UF de notificação } & \multicolumn{2}{|c|}{2016} & \multicolumn{2}{|c|}{2017} & \multicolumn{2}{|c|}{2018} & \multicolumn{2}{|c|}{2019} & \multicolumn{2}{|c|}{2020} & \multicolumn{2}{|c|}{ Total } \\
\hline & $\mathrm{N}$ & $\%$ & $\mathrm{~N}$ & $\%$ & $\mathrm{~N}$ & $\%$ & $\mathrm{~N}$ & $\%$ & $\mathrm{~N}$ & $\%$ & $\mathrm{~N}$ & $\%$ \\
\hline Mato Grosso & 3466 & 10,76 & 4423 & 12,76 & 5849 & 15,82 & 5620 & 15,53 & 2795 & 14,35 & 22153 & 13,89 \\
\hline Maranhão & 4227 & 13,12 & 4103 & 11,84 & 4155 & 11,24 & 4236 & 11,70 & 2308 & 11,85 & 19029 & 11,93 \\
\hline Pará & 3204 & 9,95 & 3363 & 9,70 & 3481 & 9,41 & 3496 & 9,66 & 1949 & 10,01 & 15493 & 9,71 \\
\hline Pernambuco & 2422 & 7,52 & 3183 & 9,19 & 3046 & 8,24 & 3347 & 9,25 & 1846 & 9,48 & 13844 & 8,68 \\
\hline Bahia & 2527 & 7,84 & 2829 & 8,16 & 2693 & 7,28 & 2505 & 6,92 & 1244 & 6,39 & 11798 & 7,40 \\
\hline Ceará & 2026 & 6,29 & 1856 & 5,36 & 2081 & 5,63 & 1924 & 5,32 & 1214 & 6,23 & 9101 & 5,71 \\
\hline Tocantins & 1728 & 5,36 & 1682 & 4,85 & 2198 & 5,94 & 1981 & 5,47 & 1019 & 5,23 & 8608 & 5,40 \\
\hline Goiás & 1809 & 5,62 & 1752 & 5,06 & 1810 & 4,89 & 1761 & 4,87 & 985 & 5,06 & 8117 & 5,09 \\
\hline São Paulo & 1764 & 5,48 & 1603 & 4,63 & 1653 & 4,47 & 1598 & 4,42 & 812 & 4,17 & 7430 & 4,66 \\
\hline Minas Gerais & 1368 & 4,25 & 1400 & 4,04 & 1402 & 3,79 & 1469 & 4,06 & 869 & 4,46 & 6508 & 4,08 \\
\hline Piauí & 1140 & 3,54 & 1357 & 3,92 & 1318 & 3,56 & 1135 & 3,14 & 520 & 2,67 & 5470 & 3,43 \\
\hline Rio de Janeiro & 1111 & 3,45 & 1184 & 3,42 & 1187 & 3,21 & 1132 & 3,13 & 636 & 3,27 & 5250 & 3,29 \\
\hline Paraná & 742 & 2,30 & 771 & 2,22 & 732 & 1,98 & 741 & 2,05 & 445 & 2,28 & 3431 & 2,15 \\
\hline Rondônia & 563 & 1,75 & 633 & 1,83 & 878 & 2,37 & 620 & 1,71 & 406 & 2,08 & 3100 & 1,94 \\
\hline Paraíba & 548 & 1,70 & 590 & 1,70 & 643 & 1,74 & 761 & 2,10 & 357 & 1,83 & 2899 & 1,82 \\
\hline Mato Grosso do Sul & 568 & 1,76 & 553 & 1,60 & 508 & 1,37 & 652 & 1,80 & 327 & 1,68 & 2608 & 1,63 \\
\hline Amazonas & 572 & 1,78 & 558 & 1,61 & 527 & 1,43 & 523 & 1,44 & 278 & 1,43 & 2458 & 1,54 \\
\hline Espírito Santo & 512 & 1,59 & 566 & 1,63 & 569 & 1,54 & 637 & 1,76 & 91 & 0,47 & 2375 & 1,49 \\
\hline Sergipe & 372 & 1,15 & 455 & 1,31 & 426 & 1,15 & 386 & 1,07 & 284 & 1,46 & 1923 & 1,21 \\
\hline Alagoas & 326 & 1,01 & 391 & 1,13 & 449 & 1,21 & 370 & 1,02 & 225 & 1,16 & 1761 & 1,10 \\
\hline Distrito Federal & 281 & 0,87 & 257 & 0,74 & 259 & 0,70 & 292 & 0,81 & 259 & 1,33 & 1348 & 0,85 \\
\hline Rio Grande do Norte & 224 & 0,70 & 295 & 0,85 & 306 & 0,83 & 228 & 0,63 & 192 & 0,99 & 1245 & 0,78 \\
\hline Santa Catarina & 194 & 0,60 & 166 & 0,48 & 168 & 0,45 & 228 & 0,63 & 125 & 0,64 & 881 & 0,55 \\
\hline Rio Grande do Sul & 152 & 0,47 & 175 & 0,50 & 191 & 0,52 & 136 & 0,38 & 68 & 0,35 & 722 & 0,45 \\
\hline Amapá & 123 & 0,38 & 154 & 0,44 & 156 & 0,42 & 167 & 0,46 & 92 & 0,47 & 692 & 0,43 \\
\hline Acre & 137 & 0,43 & 161 & 0,46 & 154 & 0,42 & 130 & 0,36 & 86 & 0,44 & 668 & 0,42 \\
\hline Roraima & 107 & 0,33 & 194 & 0,56 & 138 & 0,37 & 119 & 0,33 & 46 & 0,24 & 604 & 0,38 \\
\hline Total & 32213 & 100 & 34654 & 100 & 36977 & 100 & 36194 & 100 & 19478 & 100 & 159516 & 100 \\
\hline
\end{tabular}

${ }^{1}$ Ano diagnóstico: 2016-2020. ${ }^{2}$ Ano Notificação: 2016-2020. Fonte: Ministério da Saúde/SVS - DASIS - Sistema de Informações sobre Nascidos Vivos (SINASC) (2021).

Conforme dados retirados do Boletim Epidemiológico de Hanseníase de 2020, do Ministério da Saúde, foram reportados à Organização Mundial de Saúde (OMS) 208.619 novos casos da doença, em 2018. Dentre esses casos, quase 31.000 ocorreram nas Américas, sendo que 28.660 desses (92,6\% dos casos das Américas) foram notificados no Brasil. Dito isso, a Hanseníase é uma patologia de elevada magnitude no Brasil, sendo esse o país que ocupa o segundo lugar no ranking mundial de países com maior número de casos, estando apenas a Índia à sua frente (Goiabeira et al., 2018). O Brasil ainda apresenta uma alta endemicidade em grande quantidade de unidades federativas e cidades, o que indica uma fonte de transmissibilidade contínua, a persistência do bacilo na comunidade e dificuldades no controle e vigilância da doença nessas localidades (Schneider \& Freitas, 2018).

Em consonância com os dados do SINASC, apresentados na tabela 1, um estudo quantitativo descritivo do perfil clínico e epidemiológico da Hanseníase, apontou que há um coeficiente muito elevado da doença nas regiões Nordeste, Centro-Oeste e Norte quando comparadas às regiões Sudeste e Sul (Goiabeira et al., 2018). Tal fato, condiz com a relação da doença com indicadores de pobreza como baixas renda e escolaridade, sendo o Nordeste reconhecido entre as regiões menos desenvolvidas, ao contrário das regiões Sudeste e Sul (Rodrigues et al.,2020). Além disso, de acordo com Bernardes et al. (2021), o Mato Grosso, 
dentre todas as unidades federativas, foi aquele com o maior número de novos casos no ano de 2019, assim como demonstrado na tabela 1 .

A prevalência de hanseníase ocorre no gênero masculino $(56,79 \%)$ e na raça parda $(59,28 \%)$, seguida pela população de raça branca $(23,37 \%)$ e raça preta $(12,48 \%)$. Quanto à escolaridade, verifica-se predomínio em indivíduos com $1^{\mathrm{a}}$ a $4^{\mathrm{a}}$ série incompleta do Ensino Fundamental (19,22\%) e $5^{\mathrm{a}}$ a $8^{\mathrm{a}}$ série incompleta do Ensino Fundamental (14,72\%), em todas as faixas etárias durante o período estudado (Tabela 2).

Tabela 2 - Número absoluto e proporção de casos de Hanseníase, segundo características epidemiológicas. Brasil, entre 2016 e 2020 .

\begin{tabular}{|c|c|c|c|c|c|c|c|c|c|}
\hline \multirow{2}{*}{ Variáveis } & & \multicolumn{2}{|c|}{0 a 14 anos } & \multicolumn{2}{|c|}{15 anos e mais } & \multicolumn{2}{|c|}{ IGN } & \multicolumn{2}{|c|}{ Total } \\
\hline & & $\mathrm{N}$ & $\%$ & $\mathrm{~N}$ & $\%$ & $\mathrm{~N}$ & $\%$ & $\mathrm{~N}$ & $\%$ \\
\hline Total & & 8607 & 100 & 150901 & 100 & 8 & 100 & 159516 & 100 \\
\hline \multicolumn{10}{|l|}{ Gênero } \\
\hline & Masculino & 4542 & 52,77 & 86050 & 57,02 & 3 & 37,50 & 90595 & 56,79 \\
\hline & Feminino & 4065 & 47,23 & 64839 & 42,98 & 5 & 62,50 & 68909 & 43,20 \\
\hline & Ignorado & - & & 11 & & - & & 11 & 0,01 \\
\hline \multicolumn{10}{|l|}{ Raça } \\
\hline & Parda & 5887 & 68,40 & 88672 & 58,76 & 4 & 50,00 & 94563 & 59,28 \\
\hline & Branca & 1308 & 15,20 & 35968 & 23,84 & 3 & 37,50 & 37279 & 23,37 \\
\hline & Preta & 976 & 11,34 & 18923 & 12,54 & 1 & 12,50 & 19900 & 12,48 \\
\hline & Ign/Branco & 302 & 3,51 & 5055 & 3,35 & - & - & 5357 & 3,36 \\
\hline & Amarela & 85 & 0,99 & 1596 & 1,06 & - & - & 1681 & 1,05 \\
\hline & Indigena & 49 & 0,57 & 687 & 0,46 & - & & 736 & 0,46 \\
\hline \multicolumn{10}{|c|}{ Escolaridade } \\
\hline & Ign/Branco/Não se aplica & 2202 & 25,58 & 28631 & 18,97 & 3 & 37,50 & 30836 & 19,33 \\
\hline & $1^{\mathrm{a}}$ a $4^{\mathrm{a}}$ série incompleta do $\mathrm{EF}$ & 1930 & 22,42 & 28727 & 19,04 & - & - & 30657 & 19,22 \\
\hline & $5^{\mathrm{a}}$ a $8^{\mathrm{a}}$ série incompleta do $\mathrm{EF}$ & 3184 & 36,99 & 20289 & 13,45 & 1 & 12,50 & 23474 & 14,72 \\
\hline & Ensino médio completo & 25 & 0,29 & 21145 & 14,01 & 1 & 12,50 & 21171 & 13,27 \\
\hline & Analfabeto & 47 & 0,55 & 13734 & 9,10 & 1 & 12,50 & 13782 & 8,64 \\
\hline & $4^{\mathrm{a}}$ série completa do $\mathrm{EF}$ & 659 & 7,66 & 10672 & 7,07 & - & - & 11331 & 7,10 \\
\hline & Ensino médio incompleto & 265 & 3,08 & 10172 & 6,74 & - & - & 10437 & 6,54 \\
\hline & Ensino fundamental completo & 295 & 3,43 & 9521 & 6,31 & 1 & 12,50 & 9817 & 6,15 \\
\hline & Educação superior completa & - & - & 5671 & 3,76 & 1 & 12,50 & 5672 & 3,56 \\
\hline & Educação superior incompleta & - & - & 2339 & 1,55 & - & - & 2339 & 1,47 \\
\hline Total & & 8607 & 100 & 150901 & 100 & 8 & 100 & 159516 & 100 \\
\hline
\end{tabular}

${ }^{1}$ Ano diagnóstico: 2016-2020. ${ }^{2}$ Ano Notificação: 2016-2020. Fonte: Ministério da Saúde/SVS - DASIS - Sistema de Informações sobre Nascidos Vivos (SINASC) (2021).

Foi constatada a prevalência no gênero masculino durante esse período em todas as faixas etárias. Esse fato se justifica pela maior exposição dos homens aos fatores desencadeantes da doença em seus locais de trabalho (Goiabeira et al., 2018). Além disso, os homens desenvolvem resposta imunológica menor ao M. leprae que as mulheres, o que se relaciona à maior incidência e à maior gravidade da hanseníase no gênero masculino (Basso \& Silva, 2017). Outro fator que implica na incidência, gravidade 
e consequente morbidade é a baixa procura do homem por cuidados de saúde aliada à falta de serviços específicos que atendam às suas necessidades (Pescarini et al., 2021).

Em relação à faixa etária observou-se aumento do número de casos com a idade, com um predomínio na faixa etária economicamente ativa, o que pode gerar um impacto na economia decorrente das consequências da doença como incapacidades físicas e deformidades, as quais podem levar ao afastamento do indivíduo de suas atividades laborais gerando custos sociais adicionais (Goiabeira et al., 2018). O aumento relativo de casos novos na população de idosos $\left(60^{+}\right)$está associado à diminuição da transmissão da doença. Já no grupo menor de 15 anos significa persistência de transmissão ativa (Rocha et al., 2020).É importante o acompanhamento dos casos em menores de 15 anos pois sinaliza a existência de focos ativos de transmissão, exposição precoce à Mycobacterium leprae e infecção recente ( Souza et al., 2028). Sobre a faixa etária de 40 a mais de 60 anos, a alta frequência pode ser justificada pelo grande período de incubação da doença e pelo maior contato social dos indivíduos, assim há maior chance de entrar em um episódio de contágio (Silva et al., 2020).

Destaca-se predomínio da população parda em todas as faixas etárias, seguida pela população de raça branca e depois de raça preta. Isso ocorre provavelmente devido à cor parda ser a etnia predominante do Brasil e devido à miscigenação e à autoidentificação da população. Logo, não existe relação entre a pigmentação da pele e a possibilidade de contágio da hanseníase (Silva et al., 2020).

No que tange à escolaridade, há um maior número de casos entre indivíduos que possuem ensino fundamental incompleto (33,94\%), o qual reflete a falta de conhecimento sobre a doença e a demora na procura pelo serviço de saúde, fatores que prejudicam o diagnóstico precoce e a adesão ao tratamento (Basso \& Silva, 2017). Nesse aspecto, é importante ressaltar a relação da baixa escolaridade com a baixa renda, sendo então a hanseníase uma doença influenciada por fatores socioeconômicos. Segundo Soares et al. (2021), quanto menor o nível de escolaridade, maior o contexto de vulnerabilidade e de negligência. Dessa forma, a hanseníase corresponde a um agravo infeccioso frequente em situações de pobreza, colaborando para a manutenção da desigualdade (Campos et al., 2018).

Como pode ser observado, de acordo com a Tabela 3, houve prevalência, em todas as faixas etárias do período estudado, da classe operacional multibacilar, com 79,09 \% dos casos, seguida pela paubacilar com 20,81\%, das formas clínicas multibacilares dimorfas $(51,07 \%)$ e vichovianas $(17,30 \%)$; do grau de incapacidade $0(52,22 \%)$ e da cura $(58,22 \%)$, como tipo de saída. 
Tabela 3 - Número absoluto e proporção de casos de Hanseníase, segundo características clínicas, por faixa etária. Brasil, entre 2016 e 2020.

\begin{tabular}{|c|c|c|c|c|c|c|c|c|c|}
\hline \multirow{2}{*}{ Variáveis } & & \multicolumn{2}{|c|}{0 a 14 anos } & \multicolumn{2}{|c|}{15 anos e mais } & \multicolumn{2}{|c|}{ IGN } & \multicolumn{2}{|c|}{ Total } \\
\hline & & $\mathrm{N}$ & $\%$ & $\mathrm{~N}$ & $\%$ & $\mathrm{~N}$ & $\%$ & $\mathrm{~N}$ & $\%$ \\
\hline Total & & 8607 & 100 & 150901 & 100 & 8 & 100 & 159516 & 100 \\
\hline \multicolumn{10}{|c|}{ Classe Operacional } \\
\hline & Multibacilar & 5150 & 59,84 & 121008 & 80,19 & 6 & 75,00 & 126164 & 79,09 \\
\hline & Paucibacilar & 3448 & 40,06 & 29740 & 19,71 & 2 & 25,00 & 33190 & 20,81 \\
\hline & $\operatorname{Ign} /$ Branco & 9 & 0,10 & 153 & 0,10 & - & - & 162 & 0,10 \\
\hline \multicolumn{10}{|l|}{ Forma Clínica } \\
\hline & Dimorfa & 3951 & 45,90 & 77506 & 51,36 & 2 & 25,00 & 81459 & 51,07 \\
\hline & Virchowiana & 568 & 6,60 & 27030 & 17,91 & 1 & 12,50 & 27599 & 17,30 \\
\hline & Tuberculóide & 1595 & 18,53 & 16281 & 10,79 & 3 & 37,50 & 17879 & 11,21 \\
\hline & Indeterminada & 1814 & 21,08 & 15617 & 10,35 & - & - & 17431 & 10,93 \\
\hline & Ign/Branco/Não classificada & 679 & 7,89 & 14467 & 9,59 & 2 & 25,00 & 15148 & 9,49 \\
\hline \multicolumn{10}{|c|}{ Grau de Incapacidade } \\
\hline & Grau Zero & 6310 & 73,31 & 76980 & 51,01 & 4 & 50,00 & 83294 & 52,22 \\
\hline & Grau I & 1093 & 12,70 & 39398 & 26,11 & - & - & 40491 & 25,38 \\
\hline & Em Branco/Não avaliado & 944 & 10,97 & 20710 & 13,72 & 3 & 37,5 & 21657 & 13,58 \\
\hline & Grau II & 260 & 3,02 & 13813 & 9,15 & 1 & 12,50 & 14074 & 8,82 \\
\hline \multicolumn{10}{|l|}{ Tipo de Saída } \\
\hline & Cura & 5588 & 64,92 & 88171 & 58,43 & 2 & 25,00 & 93761 & 58,78 \\
\hline & Não preenchido & 1701 & 19,76 & 37002 & 24,52 & 5 & 62,50 & 38708 & 24,27 \\
\hline & Transf. para outro município & 427 & 4,96 & 7120 & 4,72 & - & - & 7547 & 4,73 \\
\hline & Abandono & 342 & 3,97 & 7964 & 5,28 & - & - & 8306 & 5,21 \\
\hline & Transf. para o mesmo município & 201 & 2,34 & 3488 & 2,31 & - & - & 3689 & 2,31 \\
\hline & Transf. para o outro estado & 148 & 1,72 & 2658 & 1,76 & - & - & 2806 & 1,76 \\
\hline & Erro diagnóstico & 189 & 2,20 & 2108 & 1,40 & - & - & 2297 & 1,44 \\
\hline & Óbito & 10 & 0,12 & 2315 & 1,53 & 1 & 12,50 & 2326 & 1,46 \\
\hline & Transf. para outro Pais & 1 & 0,01 & 75 & 0,05 & - & - & 76 & 0,05 \\
\hline
\end{tabular}

${ }^{1}$ Ano diagnóstico: 2016-2020. ${ }^{2}$ Ano Notificação: 2016-2020. Fonte: Ministério da Saúde/SVS - DASIS - Sistema de Informações sobre Nascidos Vivos (SINASC) (2021).

Sobre a classe operacional, foi constatado maior número da classe multibacilar, em todas as faixas etárias, enquanto a paubacilar foi menos frequente. Isso se justifica pelos multibacilares apresentarem grande quantidade de bacilos na derme e em mucosas e poder eliminá-los no meio exterior, assim são a principal forma de infecção (Campos et al., 2018).

O predomínio das formas clínicas multibacilares -dimorfas $(51,07 \%)$ e vichovianas $(17,30 \%)$-refletem diagnóstico tardio, pois são formas mais graves e avançadas, relacionadas com a maior chance de desenvolvimento de incapacidades físicas (Basso \& Silva, 2017). Ademais, segundo Tavares (2021), essa predominância também sugere falhas na realização do exame.

O grau de incapacidade predominante foi o grau zero $(52,22 \%)$ seguido pelo grau I $(25,38 \%)$ e grau II $(8,82 \%)$, mesmo com o alto índice de diagnóstico tardio. De acordo com o Ministério da Saúde, o grau 0 é a ausência de incapacidade, o grau I é a diminuição ou perda de sensibilidade nos olhos, mãos e/ou pés, e o grau II indica a presença de lesões e deformidades como lagoftalmo, opacidade corneana central, garras, reabsorção, mão e pé caído (Brasil, 2017). É importante destacar que a ausência de incapacidade não significa a inexistência de sintomas, pois muitos pacientes podem se queixar de dores e espessamentos dos nervos periféricos, sintomas que interferem na qualidade de vida (Silva et al., 2020). No entanto, como a maioria dos diagnósticos 
são tardios, é preciso ter atenção para uma possível negligência quanto à realização das avaliações e coleta dos dados por parte dos profissionais de saúde (Uchôa et al., 2017).

Além disso, é notório o grande número de portadores sem avaliação do grau de incapacidade (13,58\%). Este parâmetro é incluído na Estratégia Global Aprimorada e Diretrizes Operacionais da Organização Mundial de Saúde (OMS), o qual constitui um indicador de qualidade que retrata a subnotificação e diagnóstico tardio dos casos (Araújo et al., 2017).

Em relação ao tipo de saída, a maioria dos casos, em todas as faixas etárias, são curados e não preenchidos. Esse dado de cura reflete o acompanhamento íntimo e longitudinal oferecido pelas equipes de saúde da família e ações ambulatoriais desenvolvidas pela atenção básica à saúde, pois contribui para a redução das incapacidades e adesão ao tratamento (Ribeiro et al., 2018). Contudo, ainda há falhas no sistema e na capacitação dos profissionais de saúde, o que justifica o não preenchimento.

Durante esse período foi relatado a prevalência da hanseníase em mulheres não grávidas (97,2\%), e nas grávidas de acordo com a idade gestacional: $1^{\circ} \mathrm{T}(0,13 \%), 2^{\circ} \mathrm{T}(0,17 \%)$ e $3^{\circ} \mathrm{T}(0,12 \%)$, como evidencia a Tabela 4.

Tabela 4 - Relação dos números absolutos e proporções de casos de Hanseníase, pela idade gestacional e ano de diagnóstico dos casos de Hanseníase no Brasil, entre 2016 e 2020.

\begin{tabular}{ccccccccccccccc}
\hline \multirow{2}{*}{ Gestante } & \multicolumn{3}{c}{2016} & \multicolumn{3}{c}{2017} & \multicolumn{2}{c}{2018} & \multicolumn{2}{c}{2019} & \multicolumn{2}{c}{2020} & \multicolumn{2}{c}{ Total } \\
\cline { 2 - 14 }$y$ & $\mathrm{~N}$ & $\%$ & $\mathrm{~N}$ & $\%$ & $\mathrm{~N}$ & $\%$ & $\mathrm{~N}$ & $\%$ & $\mathrm{~N}$ & $\%$ & $\mathrm{~N}$ & $\%$ \\
\hline Não se aplica/Ign/Branco & 22081 & 68,55 & 23439 & 67,64 & 24588 & 66,5 & 24209 & 66,89 & 13353 & 68,56 & 107670 & 67,5 \\
Não & 9967 & 30,94 & 11018 & 31,79 & 12172 & 32,92 & 11818 & 32,65 & 6024 & 30,93 & 50999 & 31,97 \\
$2^{\circ}$ Trimestre & 50 & 0,16 & 64 & 0,18 & 74 & 0,20 & 52 & 0,14 & 31 & 0,16 & 271 & 0,17 \\
$1^{\circ}$ Trimestre & 38 & 0,12 & 46 & 0,13 & 53 & 0,14 & 43 & 0,12 & 20 & 0,10 & 200 & 0,13 \\
Idade gestacional ignorada & 33 & 0,10 & 50 & 0,14 & 47 & 0,13 & 37 & 0,10 & 21 & 0,11 & 188 & 0,12 \\
$3^{\circ}$ Trimestre & 44 & 0,14 & 37 & 0,11 & 43 & 0,12 & 35 & 0,10 & 29 & 0,15 & 188 & 0,12 \\
Total & 32213 & 100 & 34654 & 100 & 36977 & 100 & 36194 & 100 & 19478 & 100 & 159516 & 100 \\
\hline
\end{tabular}

${ }^{1}$ Ano diagnóstico: 2016-2020. ${ }^{2}$ Ano Notificação: 2016-2020. Fonte: Ministério da Saúde/SVS - DASIS - Sistema de Informações sobre Nascidos Vivos (SINASC) (2021).

É necessário salientar que, de acordo com o Ministério da Saúde, o surgimento dos primeiros sinais ou agravamento da doença começam na gravidez, por isso muitos casos podem ser diagnosticados nesse período. Além disso, o bebê corre o risco de nascer prematuro e com baixo peso, além de poder apresentar sequelas (Brasil, 2020). Por isso, mulheres em idade fértil e com vida sexual ativa recebem a recomendação de adotar métodos contraceptivos até que a doença seja curada, pois o recomendado é que a mulher evite engravidar no período em que está fazendo o tratamento (Nogueira et al., 2014).

\section{Conclusão}

Dado o exposto, constatamos que a hanseníase ainda é um problema de saúde pública no país, visto que o Brasil é o maior responsável por casos da doença na América Latina, destacando-se as regiões Nordeste, Norte e Centro-Oeste como as principais produtoras de novos casos. Em acréscimo, concluímos que o gênero masculino é o mais acometido pela doença, já que ele desenvolve uma resposta imunológica menor quando comparado ao gênero feminino, afetando principalmente indivíduos economicamente ativos. Além disso, a falta de acesso à informação e a precariedade socioeconômica são fatores que potencializam a falta de tratamento e a disseminação da doença, com ênfase em sua forma multibacilar. Por todos esses aspectos, fica clara a necessidade da elaboração de um plano que incentive a detecção precoce e a redução das incapacidades causadas pela doença, por meio de ações de vigilância em saúde, visando o combate. Ademais, é de suma importância a adaptação da política de atenção à Hanseníase à realidade das regiões onde a incidência é maior, a fim de aumentar a adesão ao tratamento e, 
consequentemente, erradicar a doença. Logo, se faz necessário o desenvolvimento de mais estudos e trabalhos que retratem a situação atual epidemiológica da hanseníase no Brasil.

\section{Referências}

Araújo, R. M d. S., Tavares, C.M., Silva, J.M. d.O., Alves, R. d. S., Santos, W. B., Rodrigues P.L. d. S. (2017). Análise do Perfil Epidemiológico da Hanseníase. Revista de Enfermagem UFPE online, 11,3632-3641. DOI: 10.5205/ reuol.10620-94529-1-SM.1109sup201717.

Basso, M.E.M, \& Silva, L. R.F. (2017). Perfil clínico-epidemiológico de pacientes acometidos pela hanseníase atendidos em uma unidade de referência. Revista da Sociedade Brasileira de Clínica Médica, 15 (1),27-32.

Brasil. Ministério da Saúde. Secretaria de Vigilância em Saúde. (2020). Hanseníase. Boletim Epidemiológico. 1. ed.

Brasil. Ministério da Saúde. Secretaria de Vigilância em Saúde. Departamento de Vigilância das Doenças Transmissíveis. (2017). Guia prático sobre a hanseníase [recurso eletrônico]. Brasília.

Bernardes, M. P., Oliveira, G. S., Grattapaglia, R. P. A., Melo, J. O., França, C. W., \& Pereira, G. M. (2021). Análise do Perfil Epidemiológico de Hanseníase no Brasil no período de 2010 a 2019. Brazilian Journal Of Health Review, 4 (6), 23692-23699.

Campos, M. R. M., Batista, A. V. A., \& Guerreiro, J. V.(2018). Perfil Clínico-Epidemiológico dos Pacientes Diagnosticados com Hanseníase na Paraíba e no Brasil, 2008 - 2012. Revista Brasileira de Ciências da Saúde, 22 (1), 79-86. DOI: 10.4034/rbcs.2018.22.01.11.

Goiabeira, Y. N. L. A., Rolim, I. L. T. P., Aquino, D. M. C., Soeiro, V. M. S., Inácio, A. S., \& Queiroz, R. C. S. (2018). Perfil epidemiológico e clínico da hanseníase em capital hiperendêmica. Revista de Enfermagem Ufpe On Line, Recife, 12 (6), 1507-1513.

Lopes, F. C., Ramos, A. C. V., Pascoal, L. M., Santos, F. S., Rollim, I. L. T. P., Serra, M. A. A. O., Santos, L. H., \& Neto, M. S. (2021). Leprosy in the context of the family health strategy in an endemic scenario in maranhão: Prevalence and associated factors. Revista Ciencia e Saude Coletiva, 26 (5), 1805-1816. DOI: 10.1590/1413-81232021265.04032021.

Nitsuma, E. N. A., Bueno, I. C., Arantes, E. O., Carvalho, A. P. M., Junior, G. F. X., Fernandes, G. R., \& Lana, F. C. F. (2021). Fatores associados ao adoecimento por hanseníase em contatos: revisão sistemática e metanálise. Revista Brasileira de Epidemiologia, 24. DOI: 10.1590/1980-549720210039.

Nogueira, P. S. F., Moreira, L.P.M., Moura, E.R.F., Gomes, A.M.A., Américo, C.F., \& Souza, S.F. (2014).Estratégia educativa voltada à gestação e anticoncepção na hanseníase. Ciência, Cuidado e Saúde, 13 (4), 634-641. DOI: 10.4025/cienccuidsaude.v13i4.17449.

Pescarini, J. M.,Teixeira, C. S. S., Silva, N. B., Sanchez, M. N., Natividade, M. S., Rodrigues, L. C., Penna, M. L. F.; Barreto, M. L., Brickley, E. B., Penna, G. O., \& Nery, J. S. (2021). Epidemiological characteristics and temporal trends of new leprosy cases in Brazil: 2006 to 2017. Cadernos de Saude Publica, 37 (7). DOI: 10.1590/0102-311X00130020.

Ploemacher, T., Faber, W. R., Menke, H., Rutten, V., \& Pieters, T.(2020). Reservoirs and transmission routes of leprosy; A systematic review. PLoS Neglected Tropical Diseases, 14 (4), 1-27. DOI: 10.1371/journal.pntd.0008276..

Ribeiro, M. D., Silva, J. C., \& Oliveira, S. (2018). Estudo epidemiológico da hanseníase no Brasil: reflexão sobre as metas de eliminação. Revista Panamericana de Salud Pública, 1-7. DOI: 10.26633/rpsp.2018.42.

Rocha, M. C. N., Nobre, M. L., \& Garcia, L.P. (2020). Características epidemiológicas da hanseníase nos idosos e comparação com outros grupos etários, Brasil (2016-2018). Cadernos de Saúde Pública, 36 (9). DOI: 10.1590/0102/311X00048019.

Rodrigues, R. N., Leano, H. A d. M., Bueno, I. de C., Araújo, K.M d. F.A., \& Lana, F. C .F. (2020). Áreas de alto risco de hansení ase no Brasil, perí odo 20012015. Revista Brasileira de Enfermagem, 73(3). DOI:10.1590/0034-7167-2018-0583.

Santos, Á. N., Costa, A. K. A. N., Souza, J. É. R. d., Alves, K. A. N., Oliveira, K. P. M. M. d., \& Pereira, Z. B. (2020). Perfil epidemiológico e tendência da hanseníase em menores de 15 anos. Revista da Escola de Enfermagem da USP, 54. DOI: 10.1590/s1980-220x2019016803659.

Schneider, P. B., \& Freitas, B. H. B. M. d. (2018). Tendência da hanseníase em menores de 15 anos no Brasil, $2001-2016$. Cadernos de Saúde Pública, 34(3).DOI: 10.1590/0102-311x00101817.

Silva, M. D. P., Oliveira, P. T., Queiroz, A. A. R., \& Alvarenga, W.A. (2020). Hanseníase no Brasil: uma revisão integrativa sobre as características sociodemográficas e clínicas. Research, Society and Development, 9 (11). DOI: 10.33448/rsd-v9i11.10745.

Soares, G. M. M. M..,Souza, E. A., Ferreira, A.F.,Garcia, G.S. M., Oliveira, M. L. W. , Pinheiro, A. B. M..,Santos, M.A.M. \&, Ramos Junior, A. N. (2021). Fatores sociodemográficos e clínicos de casos de hanseníase associados ao desempenho da avaliação de seus contatos no Ceará, 2008-2019. Epidemiologia e Serviços de Saúde, 30 (3). DOI: 10.1590/S1679-49742021000300024.

Souza, C. D. F., Magalhães, M. A. F. M., \& Luna, C. F. (2020). Leprosy and social deprivation: Definition of priority areas in an endemic state northeastern brazil. Revista Brasileira de Epidemiologia, 23. DOI: 10.1590/1980-549720200007.

Souza, E. A. d., Ferreira, A. F., Boigny, R. N., Alencar, C. H., Heukelbach, J., Martins-Melo, F. R., Barbosa, J. C., \& Ramos Junior, A. N. (2018). Leprosy and gender in Brazil: trends in an endemic area of the Northeast region, 2001-2014. Revista de Saúde Pública, 52 (20). DOI: 10.11606/s1518-8787.2018052000335.

Tavares, A. M. R. (2021). Perfil epidemiológico da hanseníase no estado de Mato Grosso: estudo descritivo.Einstein (Sao Paulo, Brazil), 19. DOI: 10.31744/EINSTEIN_JOURNAL/2021AO5622.

Uchôa, R.E.M.N., Brito, K.K.G., Santana, E.M.F., Soares, V.L., \& Silva, M.A. Perfil clínico e incapacidades físicas em pacientes com hanseníase. Revista de Enfermagem UFPE on line, 11 (3), 1464-1472, 2017. DOI: 10.5205/1981-8963-V11I3A13990P1464-1472-2017. 